\title{
Dynamics of SVIS Model with Holling Type IV Functional Response
}

K. A. Hasan, M. F. Hama, M. B. Mrakhan

University of Sulaimani, Faculty of science and science Educations school of science-Department of Mathematics, Sulaimani, Iraq.

Kawa79b@gmail.com

University of Sulaimani, Faculty of science and science Educations school of science-Department of Mathematics, Sulaimani, Iraq.

Mudhafar.hama@univsul.edu.iq

University of Garmian, Faculty of Educations- department of Mathematics, Kalar, Iraq.

Medya.bawaxan@gmail.com

\begin{abstract}
In this paper, we will study the effect of some epidemic concepts such as immigrants and vaccine on the dynamical behaviour of epidemic models. The existence, uniqueness and boundedness of the solution are investigated. The local stability analyses of the system is carried out. The global dynamics of the system is investigated numerically.
\end{abstract}

Keywords: Continuous dynamical systems; SIS model; Vaccination; Equilibrium and stability analysis.

Mathematics Subject Classification 2000: 37N25, 91B50, 70K20.

\section{Council for InnovativResearch}

Peer Review Research Publishing System

Journal: JOURNAL OF ADVANCES IN MATHEMATICS

Vol. 11, No. 10

www.cirworld.com, editor@cirworld.com 


\section{INTRODUCTION}

Because of many infectious disease causing death and varying total population, the study of epidemic models become one of the important area in the mathematical theory of epidemiology. Epidemic model with vaccination is one of the most important models in decreasing the spread of many diseases. In [8] Kribs-Zaleta and Velasco- Hernandez presented a simple two dimensional SIS model with vaccination exhibiting backward bifurcation. Farringten [2] derived relation between vaccine efficacy against transmission and analyzed the impact of vaccination program on the transmission potential of the infection in large populations. In [3] Gumel and Moghadas proposed a model for the dynamics of an infectious disease in the presence of a preventive vaccine considering non-linear incidence rate $\frac{c I}{1+I}$. Liu et al. [4,13] proposed more realistic models that assume non-linear incidence rate given by $\frac{k I^{\ell}}{1+\alpha I^{h}}$ with $k, \ell, \alpha, h>0$. Eunha Shim [11] assumed that the total population is asymptotically constant, and supposed the incidence rate $\frac{\beta}{N}$ where $\beta$ is the transmission rate. In this paper, we will study the SVIS model with non-linear incidence rate $\frac{\beta_{2} I}{1+b I+c I^{2}}$. The effect of immigrants on the dynamical behaviour of SVIS model is considered analytically. And the sufficient condition for the existence and stability of the endemic equilibrium point is obtained. The global dynamics of the model is studied by solving it numerically for different sets of initial values and for different sets of parameters values.

\section{Model formulation}

Consider an SIS disease when a vaccination program is in effect and there is a constant flow of incoming immigrants. A population of size $N(t)$ at time $t$ is partition into three classes of individuals; susceptible, infections and vaccinated, with sizes denoted by $S(t), I(t)$ and $V(t)$, respectively which represented in the block diagram given by Fig. (1) can be represented by the following system of non-linear ordinary differential equations.

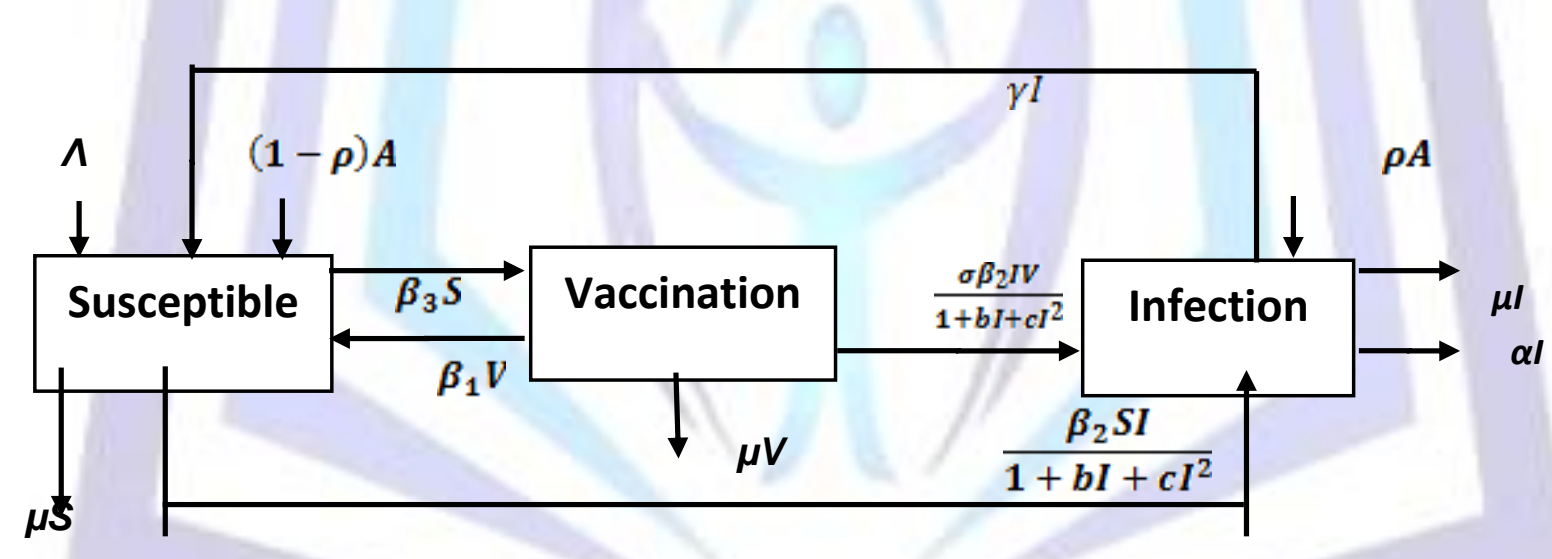

Fig 1. Model

$$
\frac{d S}{d t}=\Lambda+(1-\rho) A+\beta_{1} V+\gamma I-\frac{\beta_{2} S I}{1+b I+c I^{2}}-\mu S-\beta_{3} S
$$

$\frac{d V}{d t}=\beta_{3} S-\frac{\sigma \beta_{2} I V}{1+b I+c I^{2}}-\beta_{1} V-\mu V$

$\frac{d I}{d t}=\rho A+\frac{\beta_{2} S I}{1+b I+c I^{2}}+\frac{\sigma \beta_{2} I V}{1+b I+c I^{2}}-(\alpha+\gamma+\mu) I$

The assumption we have in this model is as follows: ${ }^{A}$ is the constant natural birth rate, with all newborns coming into the susceptible class. There is a constant flow of ${ }^{A}$ new members in to the population per unite of time, where fraction of $\rho$ with immigrants is infective $(0 \leq \rho \leq 1)$. The rate at which the vaccine wears off is $\beta_{1}, \beta_{2}$ is infection constant rate coefficient for susceptible individual and the rate at which the susceptible population is vaccinated is $\beta_{3}$. There is a 
constant per capita natural death rate ${ }^{\mu>0}$ in each class, and fraction $\gamma \geq 0$ of infectives recovers in unit time. The vaccine has the effect of reducing by a factor of ${ }^{\sigma}$, where $0 \leq \sigma \leq 1$ and $\sigma=0$ means that the vaccine is completely effective, while $\sigma=1$ means that the vaccine is totally infective. The disease can be fatal to some infectives and we define ${ }^{\alpha}$ to be the rate of disease related death. The parameters ${ }^{b, c}$ are fiting parameters of the response function, where $^{b>-2 \sqrt{c}}$ and $c>0$. Obviously, due to the biological meaning of the variables $S(t), V(t), I(t)$ system (1) has the domain $R_{+}^{3}=\left\{(\mathrm{S}, \mathrm{V}, \mathrm{I}) \in \mathfrak{R}^{3}, \mathrm{~S} \geq 0, \mathrm{~V} \geq 0, \mathrm{I} \geq 0\right.$ which is positively invariant for system(1) and all the solutions of system(1) with non-negative initial conditions are uniformly bounded as it is proved in the following theorem.

Theorem (2.1): All solution of system (1) with non-negative initial condition are uniformly bounded.

Proof: Let $(S(t), V(t), I(t))$ be any solution of the system (1) with non- negative initial condition

Since $N(t)=S(t)+V(t)+I(t)$ then $\frac{d N}{d t}=A+A-\mu(S+V+I)-\alpha I$ so $\frac{d N}{d t}+\mu N \leq A+A$

Which has an integrating factor $e^{\mu t}$ and hence a solution is $N(t)=\frac{(A+A)}{\mu}+C e^{-\mu t}$ where

$C=N(0)-\frac{(\Lambda+A)}{\mu}$ that means $N(t)=\frac{(A+A)}{\mu}\left(1-e^{-\mu t}\right)+N(0) e^{-\mu t}$. Therefore, $N(t) \leq \frac{(A+A)}{\mu}$ as $t \rightarrow \infty$, hence all solutions of system (1) that initiate in the region ${ }^{3}+$ are eventually confined in the region:

$$
B=\left\{(S, V, I): N=S+V+I=\frac{(A+A)}{\mu}\right\}
$$

Thus these solution are uniformly bounded and then the proof is complete.

3 Existence of Equilibrium point of system (1)

In this section, we find all possible equilibrium points of system (1) shows that there are at most two non-negative equilibrium points, the existence conditions for each of these equilibrium points are discussed in the following:

1) If $I=0$, then the system (1) has an equilibrium point called a disease free equilibrium point and denoted by

$\widehat{E}=(\hat{S}, \widehat{V}, 0)$, where:

$$
\hat{S}=\frac{(\Lambda+A)\left(\mu+\beta_{1}\right)}{\mu\left(\mu+\beta_{1}+\beta_{g}\right)}, \text { and } \hat{V}=\frac{\beta_{g}(\Lambda+A)}{\mu\left(\mu+\beta_{1}+\beta_{g}\right)}
$$

2) If $I \neq 0$, then the system (1) has an equilibrium point called endemic equilibrium point denoted by $E^{8}\left(S^{8}{ }_{2} V^{8}, I^{8}\right)$, where $S^{*}, V^{*}$ and $I^{*}$ represented the positive solution of the following set of equations:

$A+(1-\rho) A+\beta_{1} V+\gamma I-\frac{\beta_{2} S I}{1+b I+c I^{2}}-\mu S-\beta_{3} S=0$

$\beta_{3} S-\frac{\sigma \beta_{2} I V}{1+b I+c I^{2}}-\beta_{1} V-\mu V=0$

$\rho A+\frac{\beta_{2} S I}{1+b I+c I^{2}}+\frac{\sigma \beta_{2} I V}{1+b I+c I^{2}}-(\alpha+\gamma+\mu) I=0$

By adding Eq. (2.a), Eq. (2.b) and Eq. (2.c) we get $A+A-\mu S-\mu V-(\alpha+\mu) I=0$ that is $S^{*}=\frac{1}{\mu}\left[(\Lambda+A)-\mu\left(V^{*}+I^{*}\right)-\alpha I^{*}\right]$

Clearly $S^{*}>0$ if $0<\mu\left(V^{*}+I^{*}\right)+\alpha I^{*}<(\Lambda+A)$, from Eq.(2) we get:

$V^{*}=\frac{\beta_{3} S^{*}\left(1+b I^{*}+c I^{* 2}\right)}{\sigma \beta_{2} I^{*}+\left(\beta_{1}+\mu\right)\left(1+b I^{*}+c I^{*^{2}}\right)}$ 
Substituting the value of Eq. (3) in Eq. (4) we get:

$V^{*}=\frac{\beta_{3} G^{*}\left[D_{0}-D_{1} I^{*}\right]}{\mu\left[\sigma \beta_{2} I^{*}+D_{2} G^{*}\right]}$

Where $G^{*}=1+b I^{*}+c I^{* 2}, D_{0}=\Lambda+A_{,}, D_{1}=\mu+\alpha, D_{2}=\beta_{1}+\beta_{3}+\mu$ and $D_{3}=\alpha+\gamma+\mu$. Clearly from equation (3) we get that $V^{*}>0$.

Now by substitution Eq. (3) and Eq. (5) in Eq. (2.c) and then simplifying the resulting term gives the following polynomial equation:

$A_{5} I^{8^{5}}+A_{4} I^{8^{4}}+A_{3} I^{8^{3}}+A_{2} I^{8^{2}}+A_{1} I^{8}+A_{0}=0$

Where

$A_{5}=-c^{2} \mu D_{3} D_{2}$

$A_{4}=c^{2} \mu \rho A D_{2}-c \beta_{2} D_{1}\left(\beta_{1}+\sigma \beta_{3}+\mu\right)-c \mu D_{3}\left(2 b D_{2}+\sigma \beta_{2}\right)$

$A_{3}=c \mu \rho A\left(2 b D_{2}+\sigma \beta_{2}\right)+\beta_{2}\left(c D_{0}-b D_{1}\right)\left(\beta_{1}+\sigma \beta_{3}+\mu\right)-\mu D_{3} D_{2}\left(2 c+b^{2}\right)-$

$\sigma \beta_{2}\left(\mu b D_{3}+\beta_{2 D_{1}}\right)$

$A_{2}=2 c \mu \rho A D_{2}+\mu b \rho A\left(\sigma \beta_{2}+b D_{2}\right)+\sigma \beta_{2}^{2} D_{0}+\beta_{2}\left(b D_{0}-D_{1}\right)\left(\beta_{1}+\sigma \beta_{3}+\mu\right)-\mu D_{3}\left(2 b D_{2}+\sigma \beta_{2}\right)$

$A_{1}=\mu \rho A\left(\sigma \beta_{2}+2 b D_{2}\right)+\beta_{2} D_{0}\left(\beta_{1}+\sigma \beta_{3}+\mu\right)-\mu D_{3} D_{2}$

$A_{0}=\mu \rho A D_{2}$

Straightforward computation shows that Eq. (6) has a positive root namely $I^{8}$ provided that one set of the following sets of conditions holds

$A_{1}>0, A_{2}>0$,with $A_{3}>0$

$A_{1}<0, A_{2}<0, A_{3}<0$, with $A_{4}<0$

(7.a)

$A_{1}>0, A_{3}<0$ with $A_{4}<0$

Substitution the value of $I^{*}$ in Eq. (5) gives the value of $V^{*}$ and then substituting the value of $V^{*}$ and $I^{*}$ in Eq. (3) gives the value of $S^{8}$.

\section{Local Stability of system (1)}

In the following section the local stability analysis for the above equilibrium points is studied as shown in the following theorems.

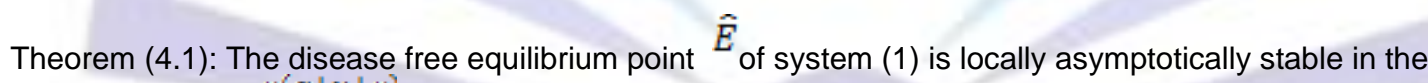

Int. $R_{+}^{3} \beta_{\text {if }}<\frac{\mu[(\alpha+\gamma+\mu])}{(\Lambda+A)}$

Proof: Therefore, the variational matrix about the equilibrium point ${ }^{\widehat{E}}$ given below:

$I(\widehat{E})=\left(\begin{array}{ccc}-\mu-\beta_{3} & \beta_{1} & \gamma-\beta_{2} \tilde{S} \\ \beta_{3} & -\beta_{1}-\mu & -\sigma \beta_{2} \hat{V} \\ 0 & 0 & \beta_{2} \hat{S}+\sigma \beta_{2} \hat{V}-(\alpha+\gamma+\mu)\end{array}\right)$

Clearly the eigenvalue of $I(\tilde{E})$ are $\lambda_{1}=-\mu<0, \lambda_{2}=-\left(\mu+\beta_{1}+\beta_{3}\right)<0$ and

$\lambda_{3}=\frac{\left(\mu+\beta_{1}+\beta_{3}\right)\left[\beta_{2}(\Lambda+A)-\mu(\alpha+\gamma+\mu)\right]+\beta_{2} \beta_{3}(\Lambda+A)(\sigma-1)}{\mu\left(\mu+\beta_{1}+\beta_{3}\right)}$

Since $0 \leq \sigma \leq 1_{\text {so }} \lambda_{3}<0$ if $\beta_{2}<\frac{\mu(\alpha+\gamma+\mu)}{(\Lambda+A)}$. 
Theorem (4.2): Assume that the positive equilibrium point $E^{*}\left(S^{*}, V^{8}, I^{8}\right)$ of the system (1) exist and let the following inequalities hold:

$c I^{*^{2}}>1$

$\theta_{12}^{2}<\theta_{11} \theta_{22}$

$\theta_{13}{ }^{2}<\theta_{11} \theta_{33}$

$\theta_{23}{ }^{2}<\theta_{22} \theta_{33}$

Here we have:

$\theta_{11}=\frac{\beta_{2} I^{8}}{1+b I^{8}+c I^{\varepsilon^{2}}}+\mu+\beta_{3} ; \theta_{12}=\beta_{1}+\beta_{3} ; \quad \theta_{22}=\frac{\sigma \beta_{2} I^{8}}{1+b I^{*}+c I^{8^{2}}}+\mu+\beta_{1}$

$\theta_{13}=\left[\frac{\beta_{2} I^{8}}{1+b I^{8}+c I^{8^{2}}}+\gamma-\frac{\beta_{2} S^{*}\left(1-c I^{8^{2}}\right)}{\left(1+b I^{*}+c I^{*^{2}}\right)^{2}}\right]$

$\theta_{23}=\frac{\sigma \beta_{2} I^{8}}{1+b I^{8}+c I^{8^{2}}}-\frac{\sigma \beta_{2} V^{8}\left(1-c I^{*^{2}}\right)}{\left(1+b I^{8}+c I^{*^{2}}\right)^{2}}$

$\theta_{33}=(\alpha+\gamma+\mu)-\frac{\beta_{2}\left(1-c I^{*^{2}}\right)\left(S^{*}+\sigma V^{*}\right)}{\left(1+b I^{8}+c I^{8^{2}}\right)^{2}}$

Then it is locally asymptotically stable in the $\operatorname{Int} R_{+}^{3}$.

Proof: The linearized system of the system (1) can be written as

$\frac{d W}{d t}=\frac{d U}{d t}=J\left(E^{*}\right) U$

Here, $W=(S, V, I)$ and $U=\left(u_{1}, u_{2}, u_{3}\right)$ with $^{u_{1}}=S-S^{8}, u_{2}=V-V^{8}$

$u_{3}=I-I^{8}$. Moreover, ${ }^{\left(E^{8}\right)}=\left(a_{i j}\right)_{3 \times 3} ; i_{j} j=1,2,3$ is the variational matrix of system (1) at $E^{8}$ in which

$a_{11}=-\frac{\beta_{2} I^{8}}{1+b I^{8}+c I^{8^{2}}}-\mu-\beta_{3}, \quad a_{12}=\beta_{1}, \quad a_{13}=\gamma-\frac{\beta_{2} S^{8}\left(1-c I^{8^{2}}\right)}{\left(1+b I^{8}+c I^{8^{2}}\right)^{2}}$

$a_{21}=\beta_{3}, \quad a_{22}=-\left[\frac{\sigma \beta_{2} I^{8}}{1+b I^{8}+c I^{8^{2}}}+\mu+\beta_{1}\right], a_{23}=-\frac{\sigma \beta_{2} V^{*}\left(1-c I^{8^{2}}\right)}{\left(1+b I^{8}+c I^{8^{2}}\right)^{2}}$

$a_{31}=\frac{\beta_{2} I^{*}}{1+b I^{*}+c I^{2}}, a_{32}=\frac{\sigma \beta_{2} I^{*}}{1+b I^{*}+c I^{*}}, a_{33}=\frac{\beta_{2}\left(1-\sigma I^{*}\right)\left(S^{*}+\sigma V^{*}\right)}{\left(1+b I^{*}+c I^{*}\right)^{2}}-(\alpha+\gamma+\mu)$

Now, consider the following function

$P=\frac{1}{2} u_{1}^{2}+\frac{1}{2} u_{2}^{2}+\frac{1}{2} u_{3}^{2}$

It is clear that $P: R_{+}^{3} \rightarrow R$ is a continuously differentiable function that satisfies that $P(0,0,0)=0$ and $P\left(u_{1}, u_{2}, u_{3}\right) \neq 0$ for all $\left(u_{1}, u_{2}, u_{3}\right) \neq(0,0,0)$

Hence $^{P}$ is a positive definite function. Now, by differentiating ${ }^{P}$ with respect to time t, gives:

$\frac{d P}{d t}=u_{1} \frac{d u_{1}}{d t}+u_{2} \frac{d u_{2}}{d t}+u_{3} \frac{d u_{3}}{d t}$ 
Substituting the value of $\frac{d u_{\tilde{i}}}{d t} ; i=1,2,3$ in the above equation, and after doing some algebraic manipulation, it gives that:$$
\begin{aligned}
\frac{d P}{d t}=-\frac{1}{2} \theta_{11} u_{1}^{2} & +\theta_{12} u_{1} u_{2}-\frac{1}{2} \theta_{22} u_{2}^{2}-\frac{1}{2} \theta_{11} u_{1}^{2}+\theta_{13} u_{1} u_{3}-\frac{1}{2} \theta_{33} u_{3}^{2}-\frac{1}{2} \theta_{22} u_{2}^{2}+\theta_{23} u_{2} u_{3} \\
& -\frac{1}{2} \theta_{33} u_{3}^{2}
\end{aligned}
$$

$\frac{d P}{d t}=-\frac{1}{2}\left(\sqrt{\theta_{11}} u_{1}-\sqrt{\theta_{22}} u_{2}\right)^{2}-\frac{1}{2}\left(\sqrt{\theta_{11}} u_{1}-\sqrt{\theta_{33}} u_{3}\right)^{2}-\frac{1}{2}\left(\sqrt{\theta_{22}} u_{2}-\sqrt{\theta_{33}} u_{3}\right)^{2}$

Obviously, due to condition (9.a)-(9.d), it is obtained that $\frac{d P}{d t}<0$, therefore the origin and then $E^{*}$ is locally asymptotically stable point in the $\ln t . R_{+}^{3}$.

\section{Globally stability of all equilibrium point}

In this section, the global dynamics of system (1) is studied with the help of Lyapunov function as shown in thefollowing theorems.

Theorem (5.1): The disease free equilibrium point $\widehat{E}$ of system (1) is globally asymptotically stable in the sub region:

$\varphi=\left\{(S, V, I): 0<I<C_{1}, \quad 0<I<K_{1}, \quad \frac{\beta_{1}}{S}+\frac{\beta_{3}}{V} \leq 2 \sqrt{\frac{\left(\mu+\beta_{1}\right)\left(\mu+\beta_{3}\right)}{S V}}\right\}$

Where

$C_{1}=\frac{-b+\sqrt{b^{2}-4 c\left(1-\frac{\beta_{I} S}{\gamma}\right)}}{2 c}$ and $K_{1}=\frac{-b+\sqrt{b^{2}-4 c\left(1-\frac{\sigma \beta_{2} \hat{V}}{(\alpha+\mu)}\right)}}{2 c}$

Proof: Consider the function $L(S, V, I)=\int_{S}^{S} \frac{u_{1}-\dot{S}}{u_{1}} d u_{1}+\int_{\vec{V}}^{V} \frac{u_{2}-\ddot{V}}{u_{2}} d u_{2}+I$

By differentiating $L$ with respect to $t$ along the solution of system (1), we get:

$\frac{d L}{d t}=\frac{S-\hat{S}}{S} \cdot \frac{d S}{d t}+\frac{V-\hat{V}}{V} \cdot \frac{d V}{d t}+\frac{d I}{d t}$

$\frac{d L}{d t}=\frac{S-\hat{S}}{S}\left[\mu \hat{S}+\beta_{3} \hat{S}-\beta_{1} \hat{V}+\beta_{1} V+\gamma I-\frac{\beta_{2} S I}{1+b I+c I^{2}}-\mu S-\beta_{3} S\right]$

$+\frac{V-\widehat{V}}{V}\left[\beta_{1} \widehat{V}+\mu \widehat{V}-\beta_{3} \hat{S}+\beta_{3} S-\frac{\sigma \beta_{2} I V}{1+b I+c I^{2}}-\beta_{1} V-\mu V\right]+\frac{\beta_{2} S I}{1+b I+c I^{2}}$

$+\frac{\sigma \beta_{2} I V}{1+b I+c I^{2}}-(\alpha+\gamma+\mu) I$

$$
\begin{gathered}
\frac{d L}{d t}=-\frac{\left(\mu+\beta_{3}\right)}{S}(S-\hat{S})^{2}-\frac{\left(\mu+\beta_{1}\right)}{V}(V-\widehat{V})^{2}+\left(\frac{\beta_{1}}{S}+\frac{\beta_{3}}{V}\right)(S-\hat{S})(V-\widehat{V})+\left(\frac{\beta_{2}}{1+b I+c I^{2}}-\frac{\gamma}{S}\right) \hat{S} I \\
+\left(\frac{\sigma \beta_{2} \hat{V}}{1+b I+c I^{2}}-(\alpha+\mu)\right) I
\end{gathered}
$$

Now for any $(S, V, I)$ in $\varphi$ and by Eq. (11) we get

$\frac{d L}{d t}<-\frac{\left(\mu+\beta_{3}\right)}{S}(S-\hat{S})^{2}-\frac{\left(\mu+\beta_{1}\right)}{V}(V-\hat{V})^{2}+\left(\frac{\beta_{1}}{S}+\frac{\beta_{3}}{V}\right)(S-\hat{S})(V-\hat{V})$

$\frac{d L}{d t}<-\frac{\left(\mu+\beta_{3}\right)}{S}(S-\hat{S})^{2}-\frac{\left(\mu+\beta_{1}\right)}{V}(V-\hat{V})^{2}+2 \sqrt{\frac{\left(\mu+\beta_{1}\right)\left(\mu+\beta_{3}\right)}{S V}}(S-\hat{S})(V-\hat{V})$ 
$\frac{d L}{d t}<-\left[\sqrt{\frac{\left(\mu+\beta_{3}\right)}{S}}(S-\hat{S})-\sqrt{\frac{\left(\mu+\beta_{1}\right)}{V}}(V-\hat{V})\right]^{2}<0$

$\frac{d L}{d t}$ is negative definite and hence $L$ is a Lyapunov function with respect to $\widehat{E}$ hence, $\widehat{E}$ is globally asymptotically stable in the sub region $\varphi$.

Theorem (5.2):Assume that the endemic equilibrium point $E^{*}\left(S^{*}, V^{*}, I^{*}\right)$ of system (1) is locally asymptotically stable then it is globally asymptotically stable in the sub region $\xi$ that satisfies the following conditions:

$\left[\left(\frac{\beta_{1}}{S}+\frac{\beta_{3}}{V}\right) G\right]^{2}<\frac{1}{S V}\left[\left(\mu+\beta_{3}\right) G+\beta_{2} I\right]\left[\left(\mu+\beta_{1}\right) G+\sigma \beta_{2} I\right]$

$\left[\frac{\gamma G}{S}-\frac{\beta_{2} S^{*} r_{2}}{S G^{*}}+\beta_{2}\right]^{2}<\frac{1}{S I}\left[\left(\mu+\beta_{3}\right) G+\beta_{2} I\right]\left[r_{1} G-\frac{\beta_{2} r_{2}\left(S^{*}+\sigma V^{*}\right)}{G^{*}}\right]$

$\left[\sigma \beta_{2}-\frac{\sigma \beta_{2} V^{*} r_{2}}{V G^{*}}\right]^{2}<\frac{1}{V I}\left[\left(\mu+\beta_{1}\right) G+++\sigma \beta_{2} I\right]\left[r_{1} G-\frac{\beta_{2} r_{2}\left(S^{*}+\sigma V^{*}\right)}{G^{*}}\right]$

Where $G=1+b I+c I^{2}, G^{*}=1+b I^{*}+c I^{* 2}, r_{1}(\alpha+\gamma+\mu)$ and $^{r_{2}}=1-c I I^{*}$

Proof: Consider the function

$F\left(S^{*}, V^{*}, I^{*}\right)=\int_{S^{*}}^{S} \frac{\tau_{1}-S^{*}}{\tau_{1}} d \tau_{1}+\int_{V^{*}}^{V} \frac{\tau_{2}-V^{*}}{\tau_{2}} d \tau_{2}+\int_{I^{*}}^{I} \frac{\tau_{3}-I^{*}}{\tau_{3}} d \tau_{3}$

By differentiating $F$ with respect to $t$ along the solution of system (1), we get:

$$
\begin{aligned}
\frac{d F}{d t}=\frac{S-S^{*}}{S}[ & \left.-\left(\mu+\beta_{3}\right)\left(S-S^{*}\right)+\beta_{1}\left(V-V^{*}\right)+\gamma\left(I-I^{*}\right)+\frac{\beta_{2} S^{*} I^{*}}{1+b I^{*}+c I^{*}}-\frac{\beta_{2} S I}{1+b I+c I^{2}}\right] \\
& +\frac{V-V^{*}}{V}\left[-\left(\mu+\beta_{1}\right)\left(V-V^{*}\right)+\beta_{3}\left(S-S^{*}\right)+\frac{\sigma \beta_{2} V^{*} I^{*}}{1+b I^{*}+c I^{*}}-\frac{\sigma \beta_{2} V I}{1+b I+c I^{2}}\right] \\
& +\frac{I-I^{*}}{I}\left[-(\alpha+\gamma+\mu)\left(I-I^{*}\right)-\frac{\beta_{2} S^{*} I^{*}}{1+b I^{*}+c I^{2}}-\frac{\sigma \beta_{2} V^{*} I^{*}}{1+b I^{8}+c I^{*}}+\frac{\beta_{2} S I}{1+b I+c I^{2}}\right. \\
& \left.+\frac{\sigma \beta_{2} V I}{1+b I+c I^{2}}\right] \\
\frac{d F}{d t}=\frac{-\left(S-S^{*}\right)^{2}}{S} & {\left[\left(\mu+\beta_{3}\right)+\frac{\beta_{2} I}{G}\right]-\frac{\left(V-V^{*}\right)^{2}}{V}\left[\left(\mu+\beta_{1}\right)+\frac{\sigma \beta_{2} I}{G}\right]+\left(S-S^{*}\right)\left(V-V^{*}\right)\left[\frac{\beta_{1}}{S}+\frac{\beta_{3}}{V}\right] } \\
& -\frac{\left(I-I^{*}\right)^{2}}{I}\left[r_{1}-\frac{\beta_{2} r_{2}\left(S^{*}+\sigma V^{*}\right)}{G G^{*}}\right]+\left(S-S^{*}\right)\left(I-I^{*}\right)\left[\frac{\gamma}{S}-\frac{\beta_{2} S^{*} r_{2}}{S G G^{*}}+\frac{\beta_{2}}{G}\right] \\
& +\left(V-V^{*}\right)\left(I-I^{*}\right)\left[\frac{\sigma \beta_{2}}{G}-\frac{\sigma \beta_{2} V^{*} r_{2}}{V G G^{*}}\right]
\end{aligned}
$$

Now from Eq. (14) and Eq. (12.a)-(12.c) we have: 


$$
\begin{gathered}
\frac{d F}{d t}=\frac{-1}{G}\left[\sqrt{\frac{\left(\mu+\beta_{3}\right) G+\beta_{2} I}{2 S}\left(S-S^{*}\right)}-\sqrt{\frac{\left(\mu+\beta_{1}\right) G+\sigma \beta_{2} I}{2 V}}\left(V-V^{*}\right)\right]^{2} \\
-\frac{1}{G}\left[\sqrt{\frac{\left(\mu+\beta_{3}\right) G+\beta_{2} I}{2 S}}\left(S-S^{*}\right)-\sqrt{\frac{r_{1} G-\frac{\beta_{2} r_{2}\left(S^{*}+\sigma V^{*}\right)}{G^{*}}}{2 I}}\left(I-I^{*}\right)\right]^{2} \\
-\frac{1}{G}\left[\sqrt{\frac{\left(\mu+\beta_{1}\right) G+\sigma \beta_{2} I}{2 V}}\left(V-V^{*}\right)-\sqrt{\frac{r_{1} G-\frac{\beta_{2} r_{2}\left(S^{*}+\sigma V^{*}\right)}{G^{*}}}{2 I}}\left(I-I^{*}\right)\right]^{2}<0
\end{gathered}
$$

So $\frac{d F}{d t}$ is negative definite and $F$ is a Lyapunov function with respect to $E^{*}$ hence, ${ }^{*}$ is globally asymptotically stable in the sub region $\xi$.

\section{Numerical analysis}

In this section the global dynamics of system (1) is studied. System (1) is solved numerically for different sets of initial conditions and different sets of parameters we choose the following set of parameter values

$\Lambda=500, \rho=0, A=100, \beta_{1}=0.12, \beta_{2}=0.00001, \beta_{3}=0.2$,

$b=2.5, c=1.5, \gamma=0.14, \mu=0.12, \sigma=0.6, \alpha=0.2$

For this parameter values, it is observed that the condition of theorem (4.1), theorem (5.1) is satisfied and trajectories with initial conditions $I_{0}=(150,200,800), I_{1}=(300,400,200), I_{2}=(200,700,800)$ converge to the disease free equilibrium point $\ddot{E}=(2727.2727,2272.727272,0)$ where all three populations coexist in the form of a stable equilibrium point. This indicates that the free equilibrium point ${ }^{\widehat{E}}$ is globally asymptotically stable. See Figure 2 .

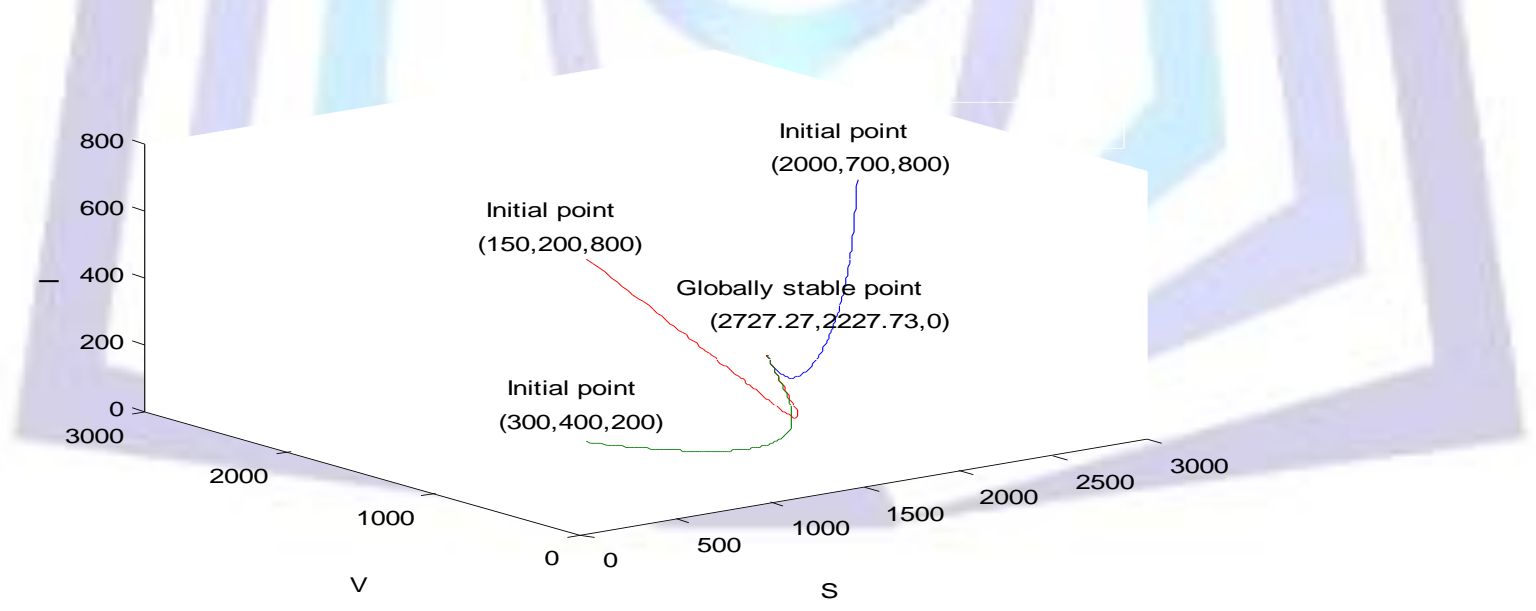

Fig 2. This figure depicts the trajectories of the model equation (15) with the initial conditions ${ }^{I_{0}, I_{1}}$ and $^{I_{2}}$. In this case, all solutions converges to the disease free equilibrium point.

Note: In the following figures, we will use the following representations: solid line for S; dashed line for $\mathrm{V}$ and dotted line for I. 


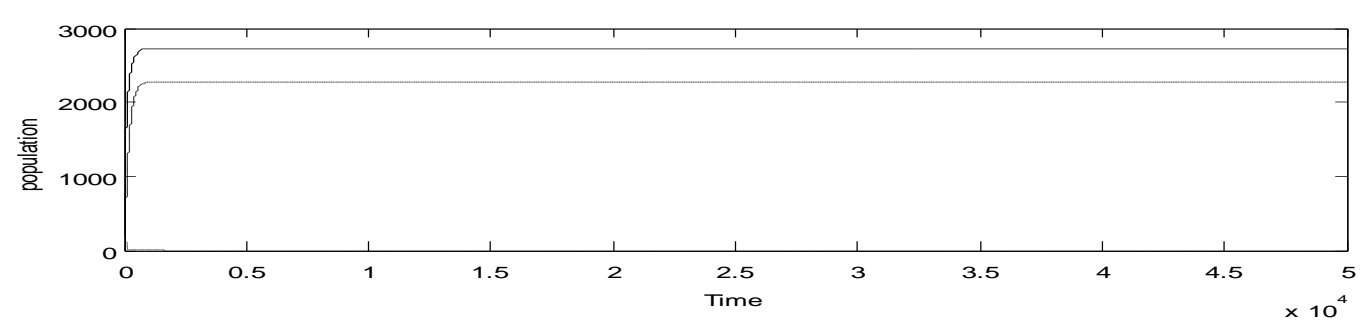

Fig 3a. Time series of trajectories of system (1) for data given in equation (15) starting at $I_{0}$

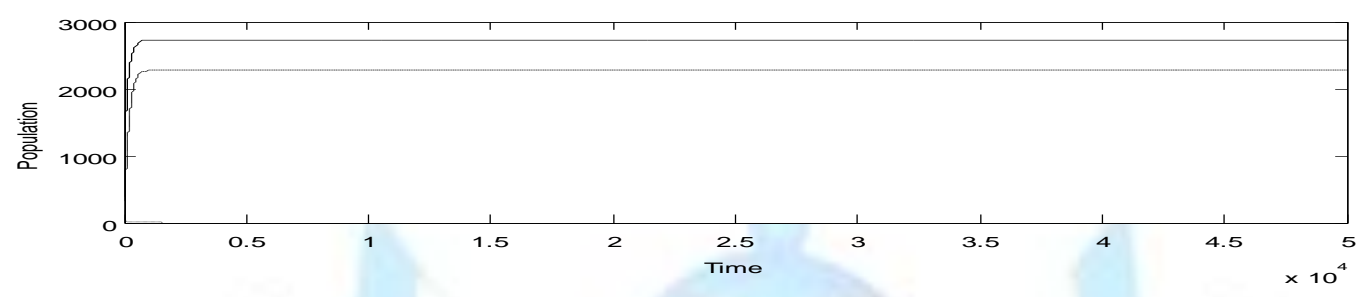

Fig 3b. Time series of trajectories of system (1) for data given in equation (15) starting at ${ }^{I_{1}}$.

However, for the set of parameters values given equation (15) with $\rho=0.2$ system (1) approaches to endemic equilibrium point $E^{*}=(2664.03,2220.02,43.4795)$ in the Int. $R_{+}^{3}$ starting from different sets of initial conditions $I_{3}=(400,2500,8), I_{4}=(50,500,10)$, and $I_{5}=(2500,700,20)$. Thus, our simulation results show that the equilibrium $E^{8}=(2664.0302022,2220.024349,43.47954323)$ is globally asymptotically stable. See Figure 4.

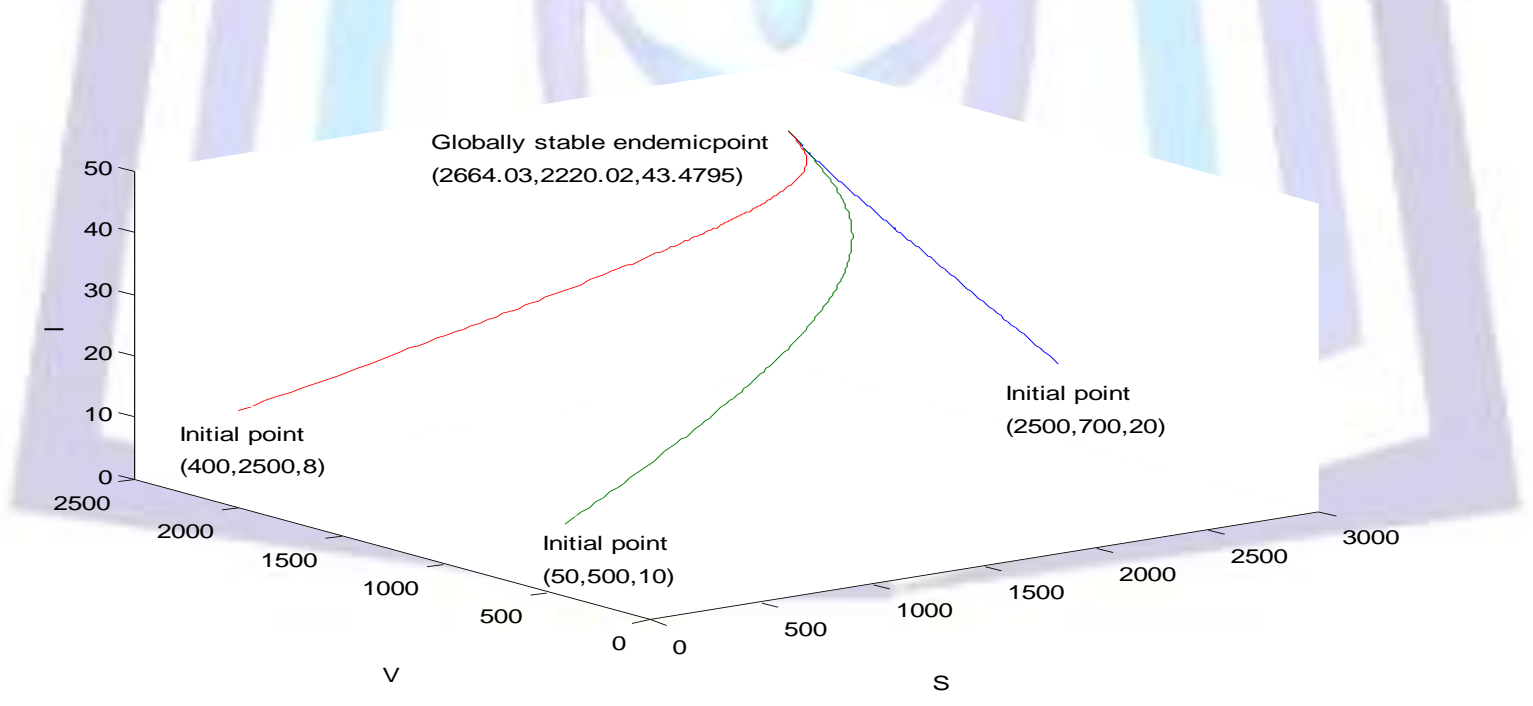

Fig 4. This figure depicts the trajectories of the model equation (15) with initial conditions ${ }^{I_{3}, I_{4}}$ and ${ }^{I_{5}}$. In this case, all solutions converges to the endemic equilibrium point $E^{*}$.

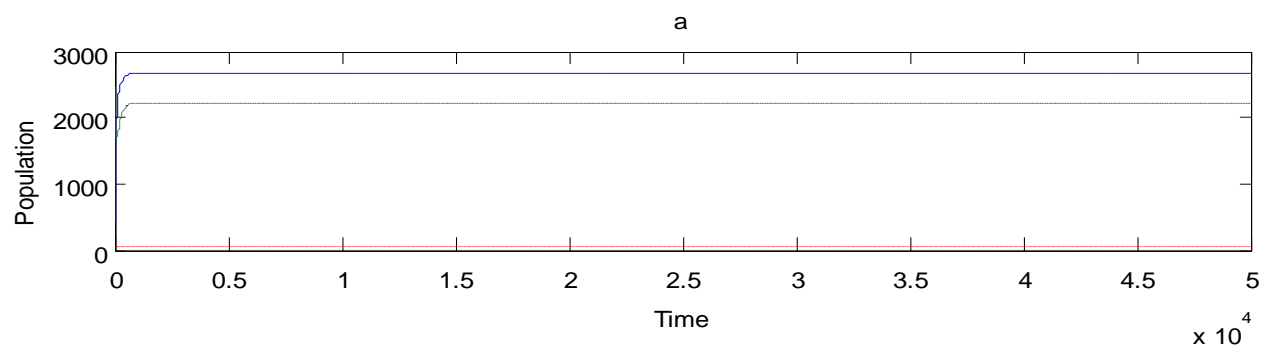




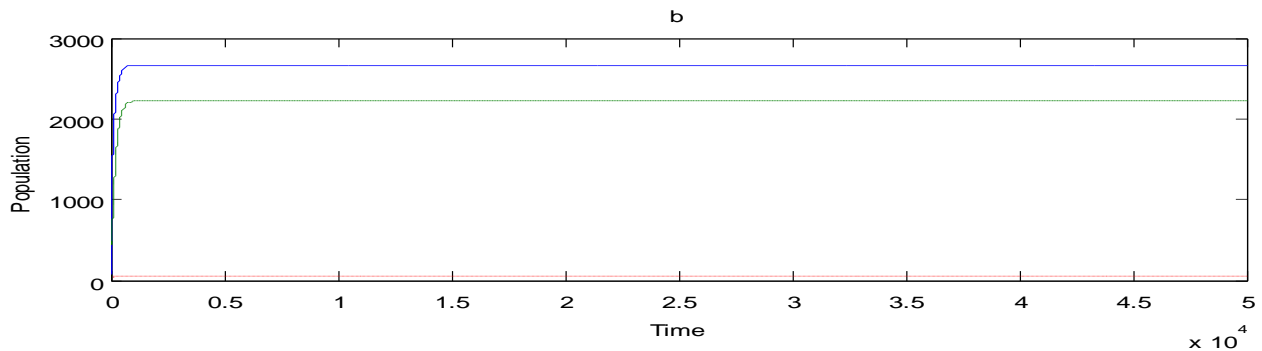

Fig 5. Time series of trajectories of system for data given in equation (15) with ${ }^{\rho=0.2}$. [a] trajectories starting at ${ }^{I_{3}}$, [b] trajectories starting at ${ }^{I_{4}}$.

Now the effect of varying the fraction of immigrant individuals, which arrive infected on the dynamics of system (1) is studied system (1) is solved for parameters values $\rho=0.05, \rho=0.4$ and $\rho=0.6$ respectively keeping other parameters fixed as given in equation (15) with $\rho=0.2$ and then the trajectories of system (1) are drawn in Figure 6.

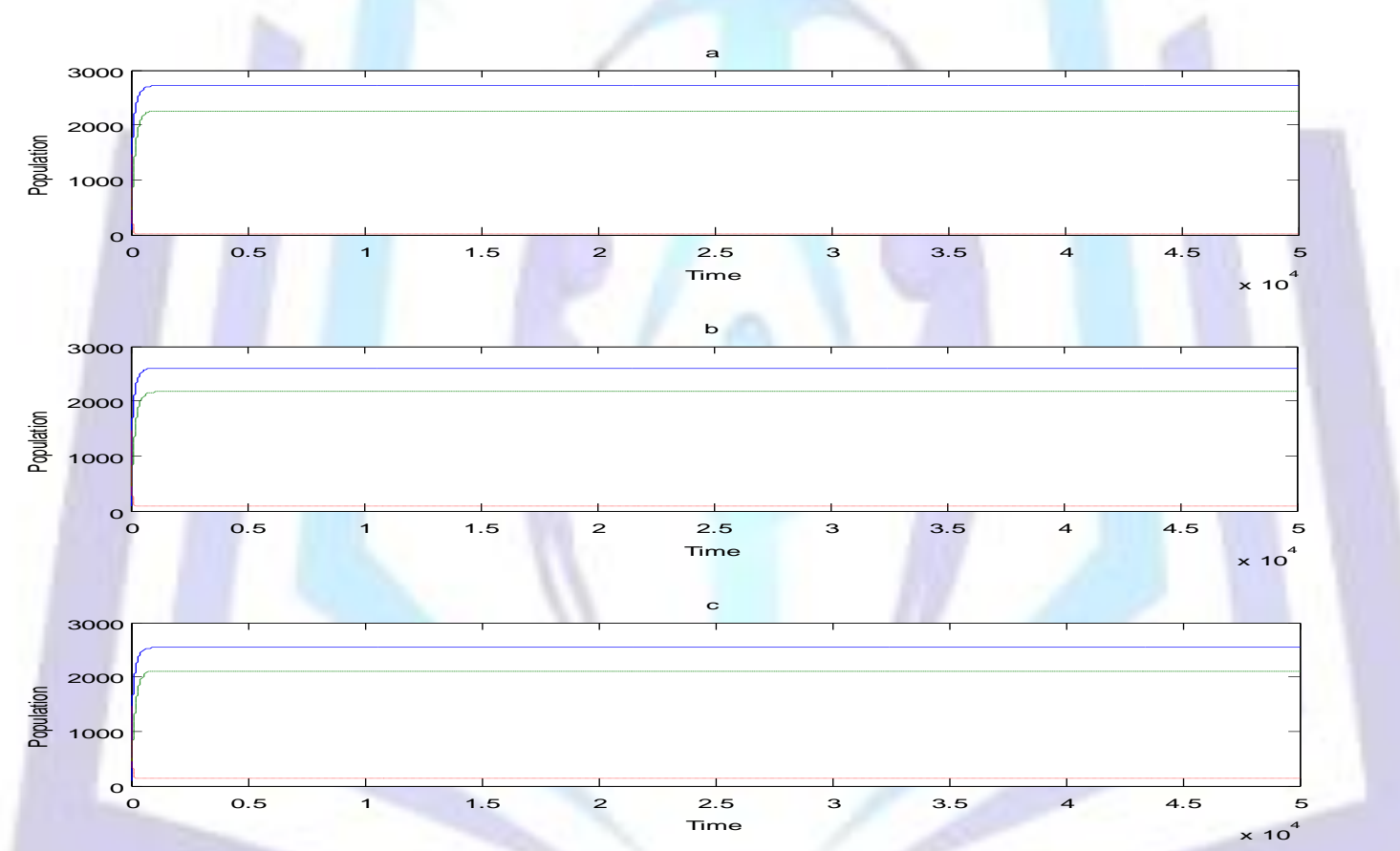

Fig 6. Time series of the solution of system (1)

a. For $\rho=0.05$ the system approaches asymptotically to $(2711.457277,2259.54474,10.87424254)$

b. For $\rho=0.4$ the system approaches asymptotically to $(2600.789808,2167.324432,86.95715964)$

c. For $\rho=0.6$ the system approaches asymptotically to $(2537.548945,2114.623854,130.4352001)$

According to Fig.(6) the disease free equilibrium point $\widehat{E}$ of system (1) becomes unstable point and the solution the system (1) approaches asymptotically to the endemic equilibrium point $E^{*}$ (where $\rho_{\text {increases). }}$

Now, in order to discuss the effect of varying the infection rate on the dynamical behavior of system (1), the system is solved for different values of infection rate $\beta_{2}=0.01, \beta_{2}=0.05$ and $\beta_{2}=0.08$ respectively, keeping other parameters fixed as given in equation (15) with $\rho=0.2$ and then the solution of system (1) is drawn in Figure 7.a-c. 

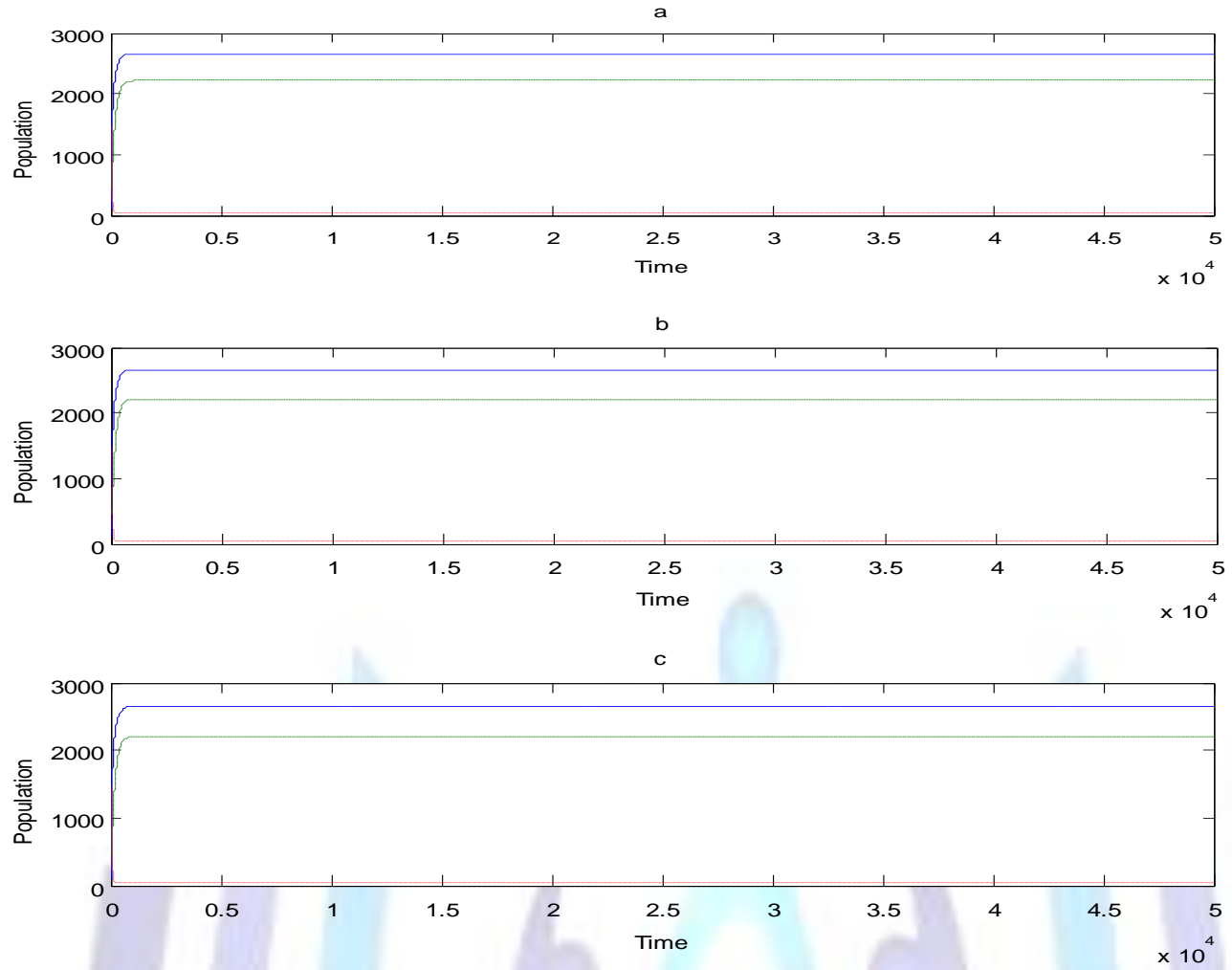

Fig 7. Time series of the solution of system (1)

a. For $\beta_{2}=0.01$ the system approaches asymptotically to $(2662.652085,2218.08013,44.7254181)$

b. For $\beta_{2}=0.05$ the system approaches asymptotically to $(2657.74771,2211.16522,49.1576489)$

c. For $\beta_{2}=0.08$ the system approaches asymptotically to $(2654.533001,2206.63609,52.6615874)$

From Figure 7.a-c we see that, if the infection rate increases the endemic equilibrium point of system (1) still coexists and stable but number of susceptible and vaccinated individuals decrease while the number of the infected individuals increases.

Also, in order to discuss the effect of varying the vaccination converge rate on the dynamical behavior of system (1) is studied too. The system is solved numerically for different value of $\beta_{3}=0.4, \beta_{3}=0.6$ and $\beta_{3}=0.8$ keeping the rest of parameters fixed as given in equation (15) with $\rho=0.2$ and time series of the solution of system (1) are drawn in Figure 8.a-c.

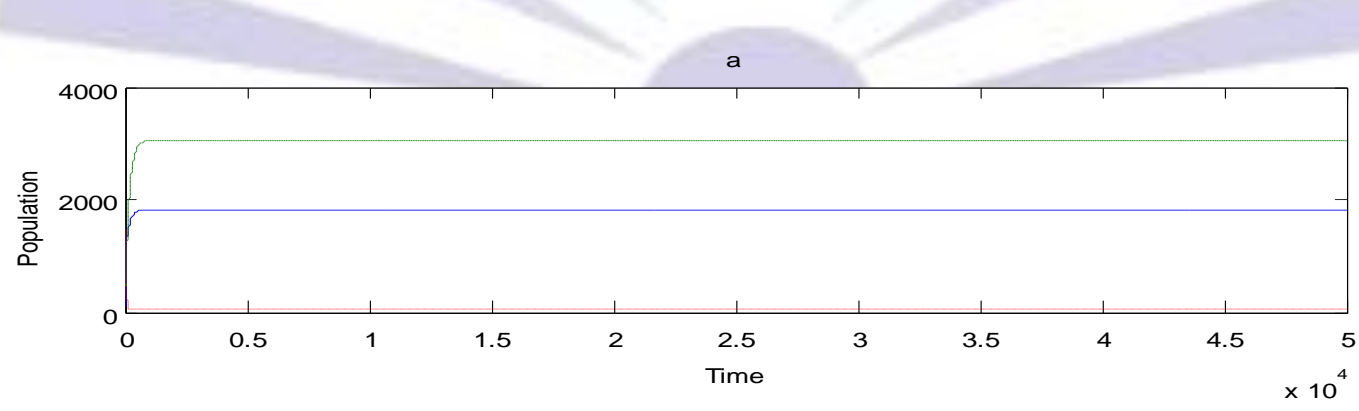



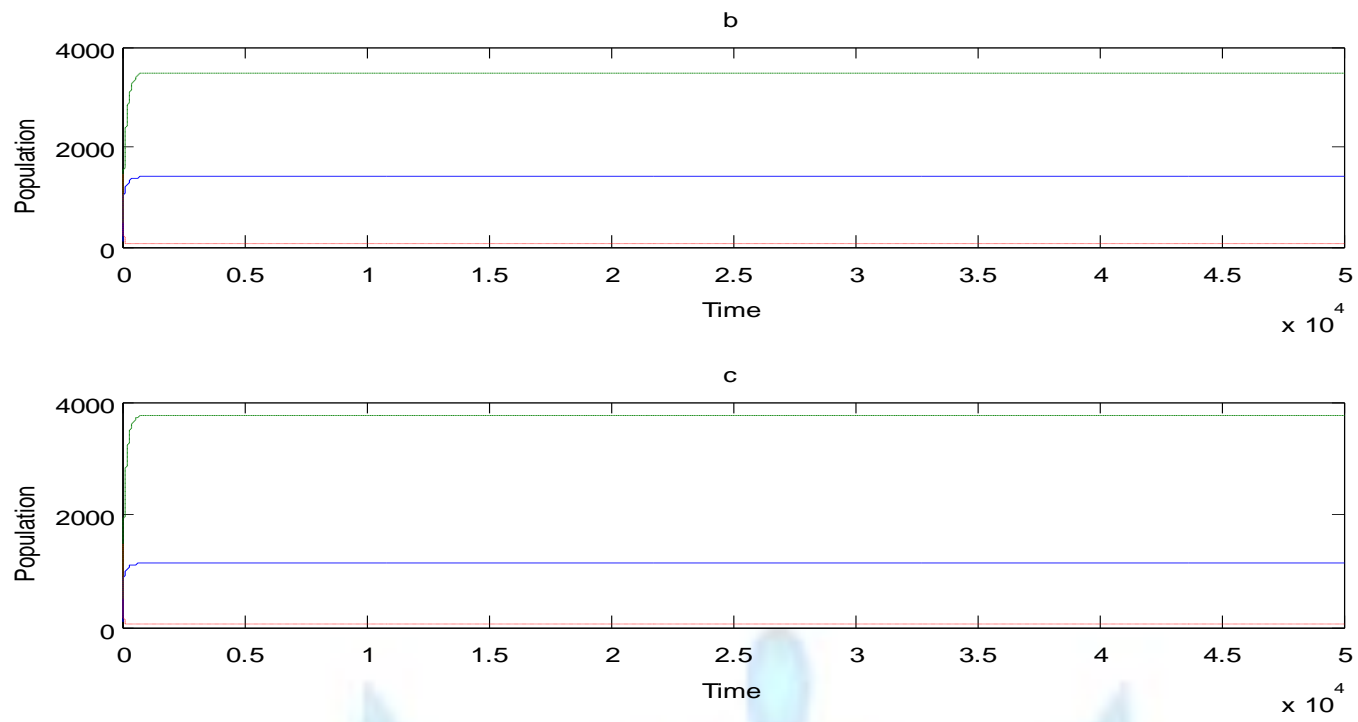

Fig 8. Time series of the solution of system (1)

a. For $\beta_{3}=0.4$ the system approaches asymptotically to $(1838.520986,3052.53385,43.4794363)$

b. For $\beta_{3}=0.6$ the system approaches asymptotically to $(1396.44464,3488.61033,43.47938)$

c. For $\beta_{3}=0.8$ the system approaches asymptotically to $(1127.089953,3756.965124,43.4793459)$

From Figure 8.a-c. we note that the system (1) still approaches to endemic equilibrium point.

Similarly, the effect of varying the number of individuals who lose vaccine immunity and return to susceptible on the dynamical behavior of system (1) is studied the system is solved for the value $\beta_{1}=0.14, \beta_{1}=0.16$ and $\beta_{1}=0.18$ keeping other parameters as given in equation (15) with $\rho=0.2$ and then the solution of system (1) are drawn in Figure 9.a-c. respectively.
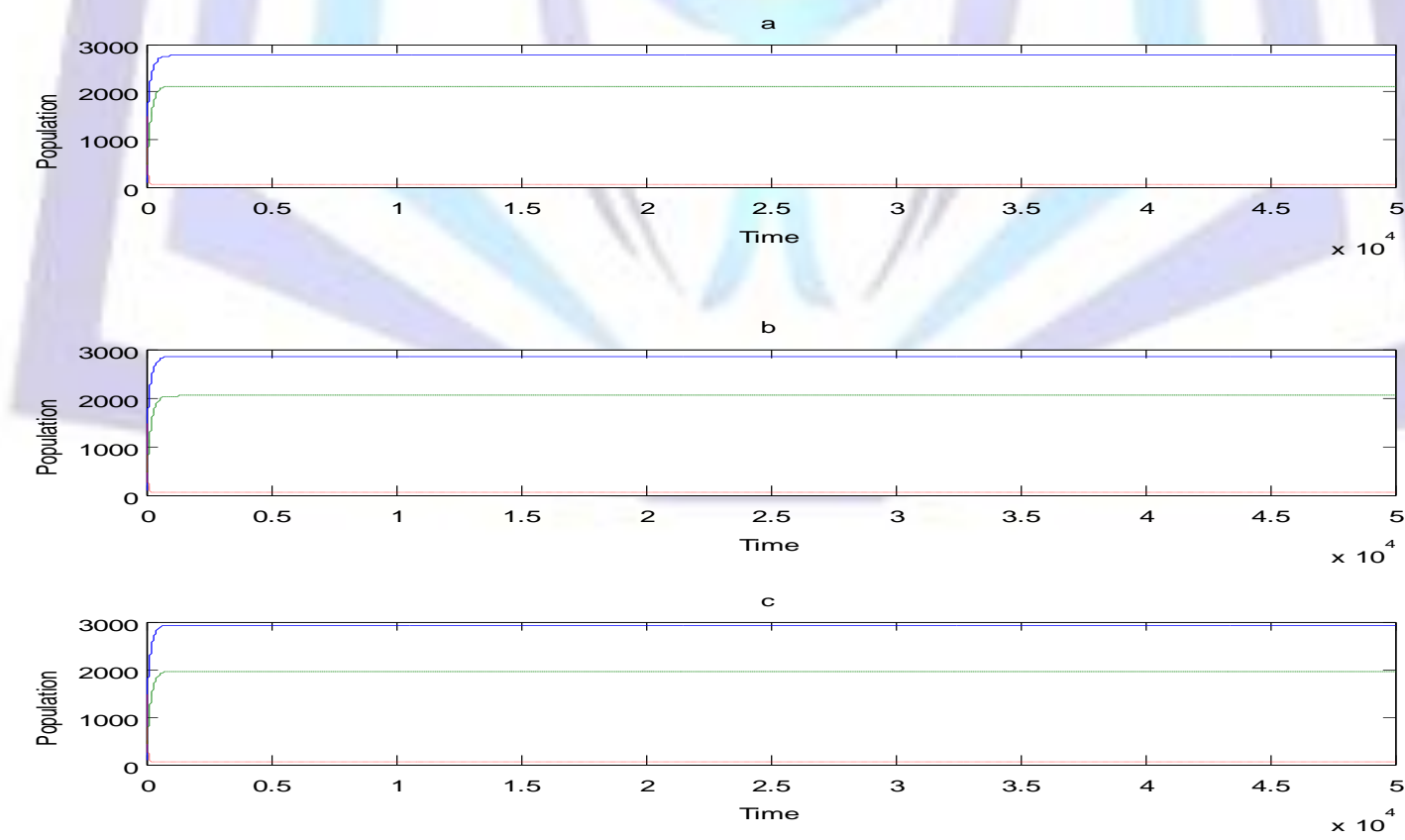

Fig 9. Time series of trajectories for system (1)
a. For $\beta_{1}=0.14$ the system approaches asymptotically to $(2760.55296,2123.50155,43.4795556)$
b. For $\beta_{1}=0.16$ the system approaches asymptotically to $(2849.03216,2035.02232,43.4795669)$
c. For $\beta_{1}=0.18$ the system approaches asymptotically to $(2930.43302,1953.62143,43.4795774)$ 
From Figure 9.a-c it is observed that as ${ }^{\beta_{1}}$ increases the system (1) still approaches to endemic equilibrium point and increasing ${ }^{\beta_{1}}$ causes increasing in the susceptible and infected but the number of vaccinated decreases.

Finally, the effect of vaccine efficiency against the disease on the dynamical behavior of system (1) is investigated. The system is solved for different values of $\sigma=0.8, \sigma=0.9$ and $\sigma=0.95$ keeping other parameters as given in equation (15) with $^{\rho=0.2}$ and then the solution of system (1) are drawn in Figure 10.a-c. respectively.
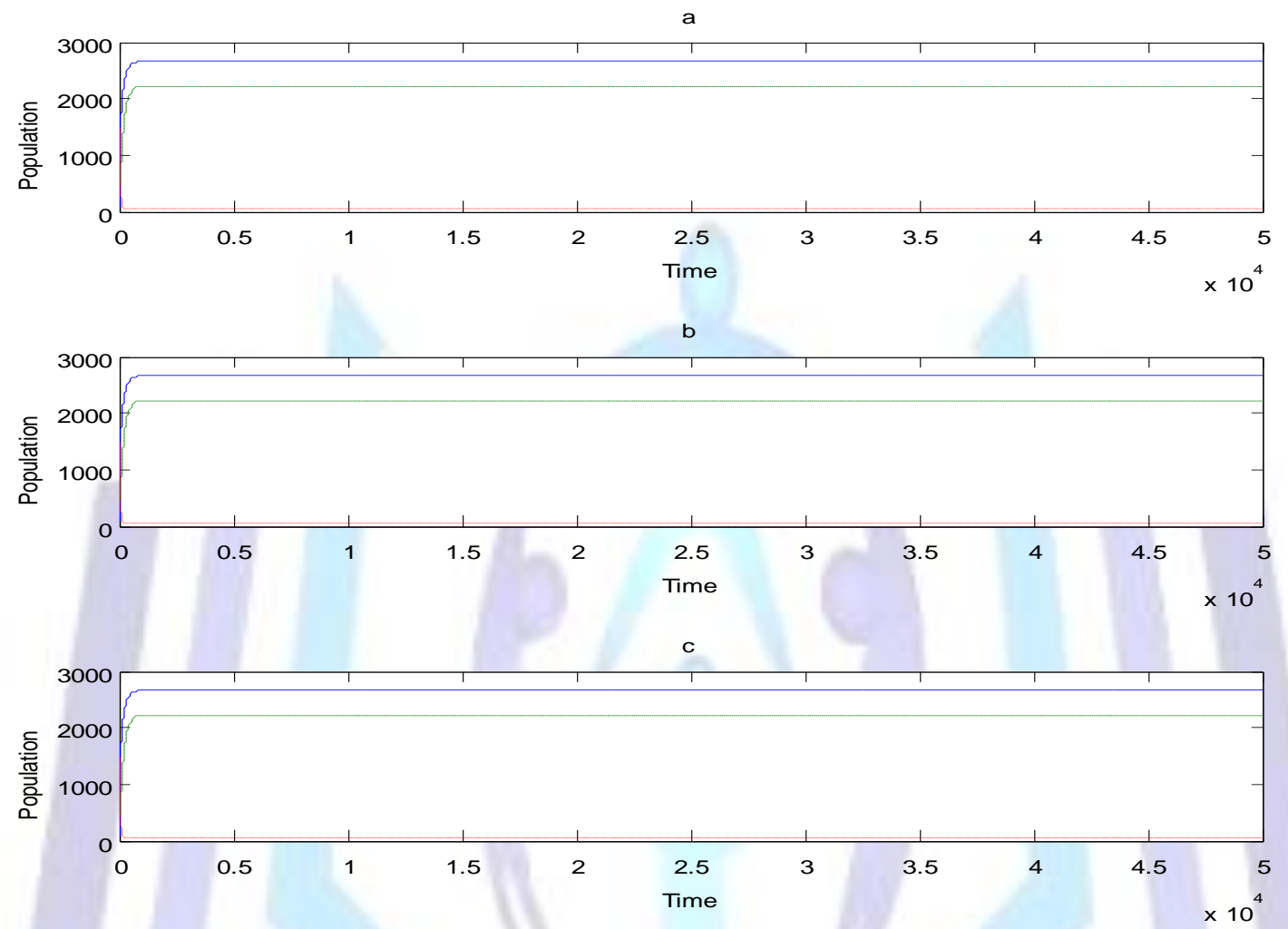

Fig 10. Time series of the solution of system (1)

a. For $\sigma=0.8$ the system approaches asymptotically to $(2664.0301439,2220.024027,43.4796857)$

b. For $\sigma=0.9$ the system approaches asymptotically to $(2664.0301148,2220.023866,43.4797569)$

c. For $\sigma=0.95$ the system approaches asymptotically to $(2664.0301002,2220.02378,43.4797925)$

From Figure 10.a-c. we conclude that as the vaccine efficiency increases the endemic equilibrium point of system (1) still coexists and stable.

\section{Discussion and conclusions}

In this section, we have analyzed a SIS epidemic model with the effect of vaccine and immigrants on the dynamical behavior on it. The local as well as global stability analysis of each possible equilibrium point are studied analytically as well as numerically. From numerically simulation (section 6) the following results are obtained the SVIS system (1) is approaches either to the disease free equilibrium point or to endemic equilibrium point.

As the fraction of infected immigrant individuals $(\rho)$ increases then the number of susceptible and vaccinated individuals increase but the number of infected individual's increases.

If the infection rate ${ }^{\left(\beta_{2}\right)}$ increases then the number of susceptible and vaccinated individuals decrease but the number of infected individual's increases increasing of ${ }^{\beta_{3}}$ causes increasing in the vaccinated but the number of susceptible infected decrease (very slowly). 
As $\beta_{1}$ increases then the number of susceptible and infected individuals increase, however the number of vaccinated individuals decreases.

Finally as the vaccine efficiency increases then the number of susceptible and vaccinated individuals decrease but the number of infected individuals increases.

\section{REFERENCES}

[1] L.Q. Gao, H.W. Hethcote. Disease models of with density-dependent emographics, J. Math. Biol. 30:717-731, 1992.

Gao and Hethcote

[2] Anderson R.M., May R.M., Population biology of infectious disease: Part I, Nature 280, 361-376, 1979.

[3] Christopher M., Jorge X., A simple vaccination model with multiple endemic states, Mathematical Biosciences. 164, 183-201, 2000.

[4] Farrington C.P ., On vaccine efficacy and reproduction numbers, Mathematical Biosciences., 185, 89-109, 2003

[5] Gumel A.B., Moghadas S.M., A qualitative study of a vaccination model with nonlinear incidence, Appl. Math. Comput., 143, 409-419, 2003.

[6] Hethcote H.W., D.W. Tudor., Integral equations models for endemic infectious disease, J. Math. Biol., 9, 37-47, 1980.

[7] Halloran M.E., Haber M., Longini I.M., Struchiner C., Direct and indirect effects in vaccine efficacy and effectiveness, Am. J. Epidemiol., 133:323-31, 1991.

[8] Kermack W.O., Mckendrick A.G, "Contributions to mathematical theory of epidemics", Proc. R. Soc. Lond. A., 115, 700$721,1927$.

[9] Kermack W. O., McKendrick A. G., Contributions to the mathematical theory of epidemics, part II. Proc. Roy. Soc. London 138, 55-83, 1932.

[10] D. Lacitignola, "Saturated treatments and measles resurgence episodes in South Africa: a possible linkage," Mathematical Biosciences and Engineering: MBE, vol. 10, no. 4, pp. 1135-1157, 2013.

[11] Eunha Shim, "A Note on Epidomic Models with Infective Immigrants and Vaccination", Mathematical Biosciences and Engineering, Vol. 3, P. 557-566, 2006.

[12] Li J., and Ma Z. "Qualitative analysis of SIS-epidemic model with vaccination and varying total population size", Math. Comput. Model, 20, 43-1235, 2002.

[13] Shulin Sun, "Global Dynamics of a SEIR Model with a Varying Total Population Size and Vaccination" Int. J. of Math. Analysis, no. 40, 1985 - 1995, 2012,

[14] Wiggins S., Introduction to applied nonlinear dynamical system and chaos, Spring-Verlag, New York, Inc., 1990.

[15] Zhou Y., Liu H. "Stability of periodic solutions for an SIS model with pulse vaccination." Math. Comput. Model. 38:299308, 2003.

[16] J. Zhou and H.W. Hethcote, Population size dependent incidence in models for diseases without immunity, J. Math. Biol. 32, 809-834,1994. 